\title{
Inferring protein domains associated with drug side effects based on drug-target interaction network
}

\author{
Hiroaki Iwata ${ }^{1 \dagger}$, Sayaka Mizutani ${ }^{2 \dagger}$, Yasuo Tabei ${ }^{3}$, Masaaki Kotera ${ }^{2}$, Susumu Goto ${ }^{2}$, Yoshihiro Yamanishi ${ }^{1,4^{*}}$ \\ From 24th International Conference on Genome Informatics (GIW 2013) \\ Singapore, Singapore. 16-18 December 2013
}

\begin{abstract}
Background: Most phenotypic effects of drugs are involved in the interactions between drugs and their target proteins, however, our knowledge about the molecular mechanism of the drug-target interactions is very limited. One of challenging issues in recent pharmaceutical science is to identify the underlying molecular features which govern drug-target interactions.

Results: In this paper, we make a systematic analysis of the correlation between drug side effects and protein domains, which we call "pharmacogenomic features," based on the drug-target interaction network. We detect drug side effects and protein domains that appear jointly in known drug-target interactions, which is made possible by using classifiers with sparse models. It is shown that the inferred pharmacogenomic features can be used for predicting potential drug-target interactions. We also discuss advantages and limitations of the pharmacogenomic features, compared with the chemogenomic features that are the associations between drug chemical substructures and protein domains.
\end{abstract}

Conclusion: The inferred side effect-domain association network is expected to be useful for estimating common drug side effects for different protein families and characteristic drug side effects for specific protein domains.

\section{Background}

Most phenotypic effects of drugs are involved in the interactions between drugs and their target proteins (drug-target interactions hereafter). Drug molecules often interact not only with a therapeutic target but also with the other proteins (off-targets hereafter), which could lead to unwanted side effects [1]. Therefore, the identification of overall target proteins of drugs including the therapeutic targets and off-targets is a crucial process in the drug development. In addition, the understanding of the molecular mechanism of drug phenotypic effects in terms of drug-target interactions is also an important issue in many pharmaceutical applications. There is a hypothesis that drug phenotypic effects are involved in many kinds of biological features of

\footnotetext{
*Correspondence: yamanishi@bioreg.kyushu-u.ac.jp

† Contributed equally

'Division of System Cohort, Multi-scale Research Center for Medical Science, Medical Institute of Bioregulation, Kyushu University, 3-1-1 Maidashi, Higashiku, Fukuoka, Fukuoka 812-8582, Japan

Full list of author information is available at the end of the article
}

drugs and proteins (e.g., drug chemical substructures, pharmacophores, protein functional sites, and biological pathways).

Recently, a variety of computational methods have been developed for large-scale prediction of drug-target interactions in the context of chemogenomics or pharmacogenomics. The key idea of the chemogenomic approach is that chemically similar compounds are likely to interact with similar proteins, and the prediction is performed based on compound chemical structures and/or protein sequences [2-7]. In contrast, the key idea of the pharmacogenomic approach is that phenotypically similar drugs are likely to interact with similar proteins, and the prediction is performed based on drug side effects and/or protein sequences [8-10]. However, the predictive models of most previous methods are not biologically interpretable, which makes it difficult to interpret biological features of drug-target interactions or compound-protein interactions. 
The identification of biological features which are associated with drug-target interactions or compoundprotein interactions is becoming a challenging issue in recent pharmaceutical science. In the context of chemogenomics, some machine learning methods with sparse models have been proposed to detect informative combinations of drug chemical substructures and protein domains which may explain the mechanism of drug-target interactions. The inferred features are called "chemogenomic features" $[11,12]$. In addition, the use of a data mining method has been proposed to infer molecular substructure pairs which appear frequently and significantly in interacting drug-target pairs [13]. The next challenge is to relate drug-target interactions with drug phenotypic effects (e.g., pharmaceutical effects and side effects). Recently, the use of drug targeted proteins has been proposed for predicting drug side effects [14-16]. The inference of proteins associated with drug side effects has been proposed $[14,17]$, but there is no previous work on the analysis at the protein domain level. Protein domains are structural, evolutional, and functional units, so it would be important to investigate the associations between protein domains and drug side effects on a large scale.

In this paper, we make a systematic analysis of the correlation between drug side effects and protein domains, which we call "pharmacogenomic features," based on the drug-target interaction network. We detect drug side effects and protein domains that appear jointly and in known drug-target interactions, which is made possible by using classifiers with sparse models. It is shown that the inferred pharmacogenomic features can be used for predicting potential drug-target interactions. We also discuss advantages and limitations of the pharmacogenomic features, compared with the chemogenomic features that are the associations between drug chemical substructures and protein domains. To our knowledge, no other computational method has been reported for relating protein domains with drug side effects. The inferred side effect-domain association network is expected to be useful for estimating common drug side effects for different protein families and characteristic drug side effects for specific protein domains.

\section{Results and discussion}

\section{Inference of pharmacogenomic features}

We applied four methods: L1LOG, L2LOG, L1LOG-C, and L2LOG-C to infer pharmacogenomic features from the drug-target interaction network. Note that L1LOG and L2LOG are respectively $L_{1}$ - and $L_{2}$-regularized logistic regressions with the tensor product descriptors, and L1LOG-C and L2LOG-C are respectively $L_{1}$ - and $L_{2}$-regularized logistic regressions with the combined descriptors (see the Methods section for more details).
In each method, we inferred pharmacogenomic features with positive weights in the predictive model.

Each pharmacogenomic feature is composed of a side effect and a protein domain which are thought of as being associated with each other. There is a tendency that the protein domain in a pharmacogenomic feature are present in the same protein family targeted by drugs causing the side effect within the corresponding pharmacogenomic feature. We quantitated degree of the associations between side effects and protein domains by evaluating the weights on the corresponding pharmacogenomic features. Figure 1 shows a small part of side effect-domain association network inferred by the L1LOG method, where edges are placed between side effects and protein domains in positively weighted pharmacogenomic features and the top 200 weights are selected in the picture because of space limitation.

We investigated the number of pharmacogenomic features inferred by the four methods. Figure 2 shows a summary of the comparison between L1LOG, L2LOG, L1LOG-C, and L2LOG-C on the gold standard data. It is found that the numbers of inferred features in L1LOG and L1LOG-C are significantly fewer than those in L2LOG and L2LOG-C, respectively. This observation means that the sparsity induced by the $L_{1}$ penalty has positive effects of reducing the number of features in the descriptors. The feature extraction property helps us to analyze the inferred features for biological interpretation in practice.

Figure 3 shows the overlap of inferred pharmacogenomic features between the four methods. It is found that L1LOG was able to infer a very limited number of features and most of the features were included in those of L2LOG. This result suggests that the inferred features of L1LOG are more representative than those of L2LOG. Both L1LOG and L1LOG-C are sparsityinduced methods, but the number of common features between the two methods was very limited. This result suggests that biological interpretation about the inferred features may depend on the descriptors designed for drug-target pairs.

\section{Reconstruction of known drug-target interactions}

We examined the validity of the pharmacogenomic features inferred by L1LOG, L2LOG, L1LOG-C, and L2LOG-C in terms of generalization properties for drug-target interactions. In order to test the ability of each method to reconstruct known drug-target interactions from the features, we performed two types of cross-validation experiments: pair-wise cross-validation and block-wise cross-validation (see the Methods section for more details). We also made a comparison between the pharmacogenomic features and the chemogenomic features. Note that the chemogenomic 


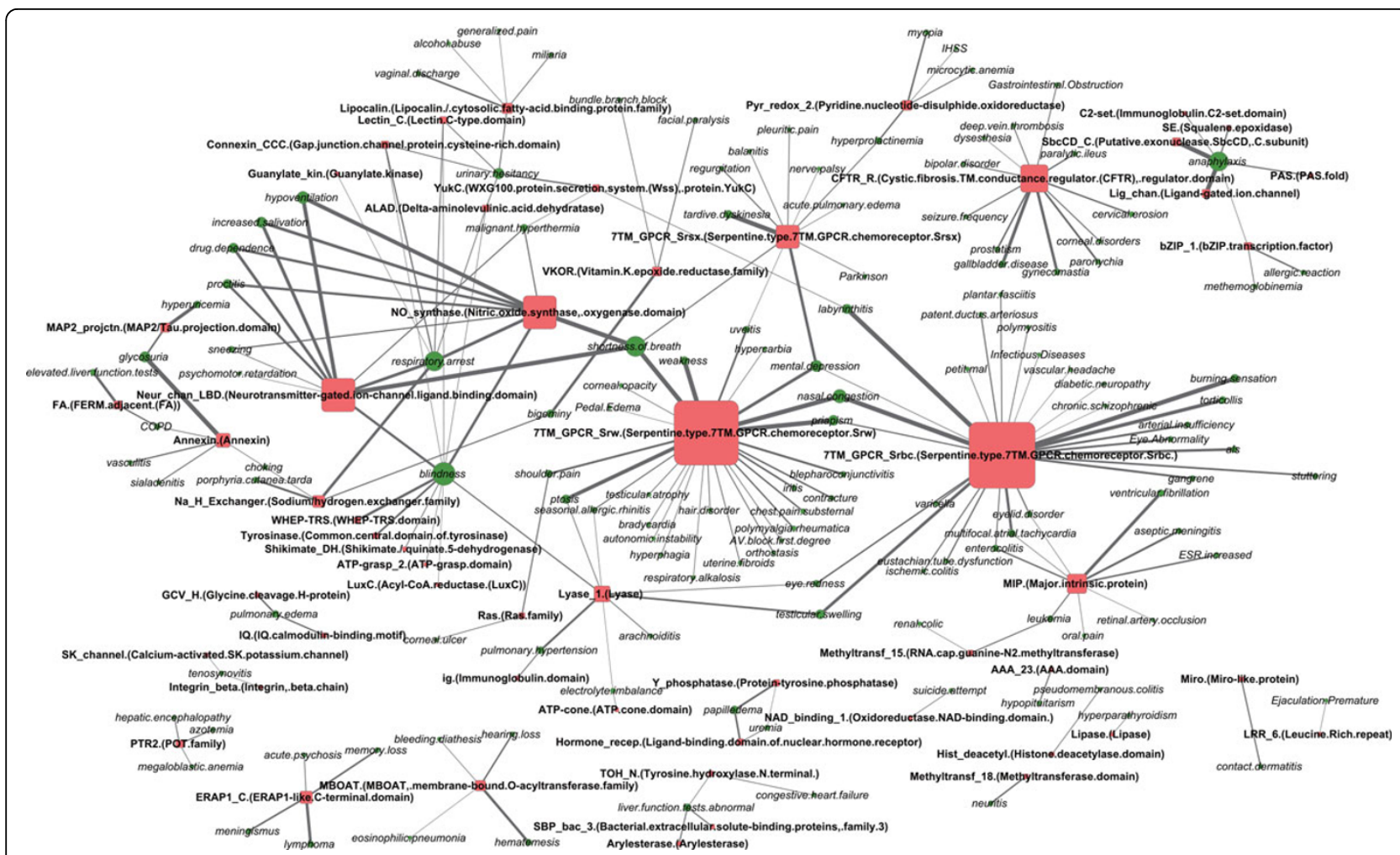

Figure 1 Part of the inferred side effect and protein domain network. Green circle and red rectangle represent a side effect and a protein domain, respectively. Node size represents a node degree. Edge width represents the weight of side effect and protein domain pair.

features correspond to the associations between drug chemical substructures and protein domains [12]. The methods with the pharmacogenomic features and the chemogenomic features are referred to as pharmacogenomic approach and chemogenomic approach, respectively, below.

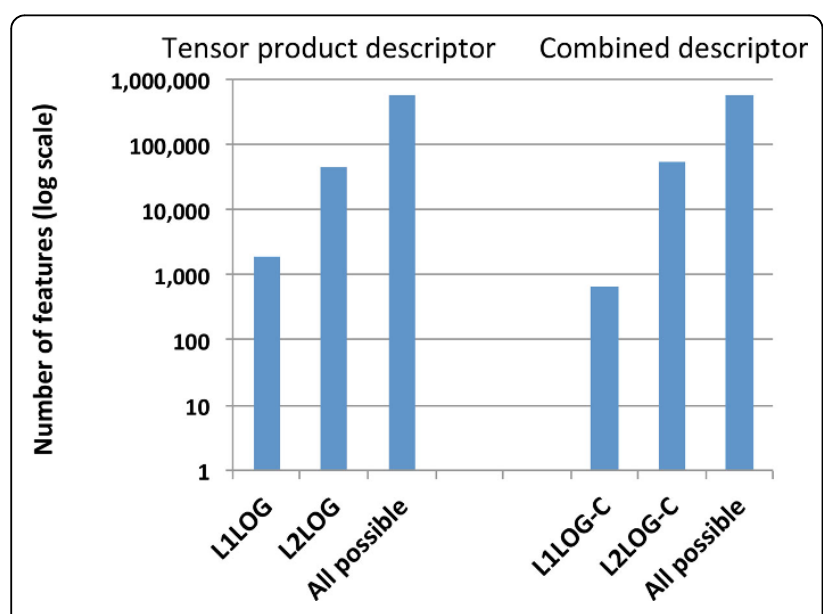

Figure 2 Barplot of the numbers of features. Comparison of the number of inferred features between L1LOG, L2LOG, L1LOG-C, and L2LOG-C.
We evaluated the performance by using the ROC curve (receiver operating characteristic curve). The ROC curve is a function of true positive rates against false positive rates based on many thresholds for the prediction scores, where true positives are correctly predicted interactions and false positives are incorrectly predicted

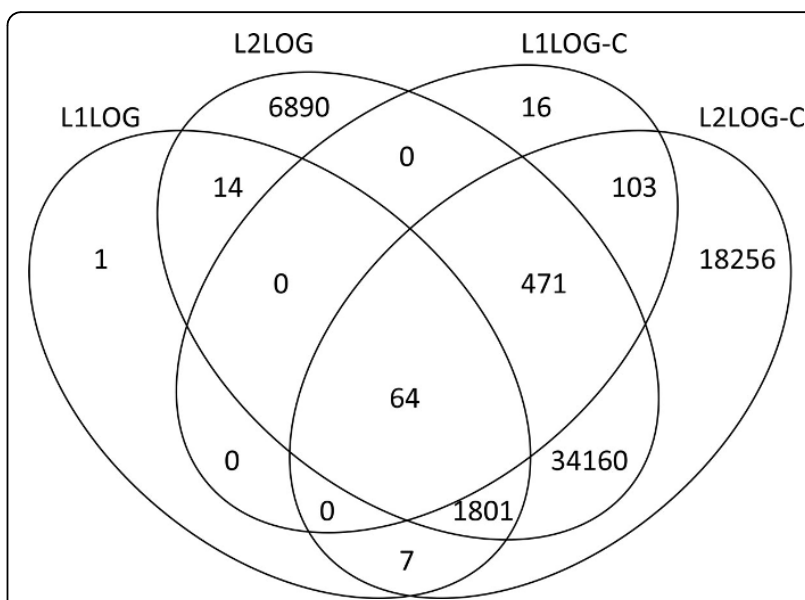

Figure 3 Overlap of the number of features. Comparison of the number of inferred features across L1LOG, L2LOG, L1LOG-C, and L2LOG-C. 


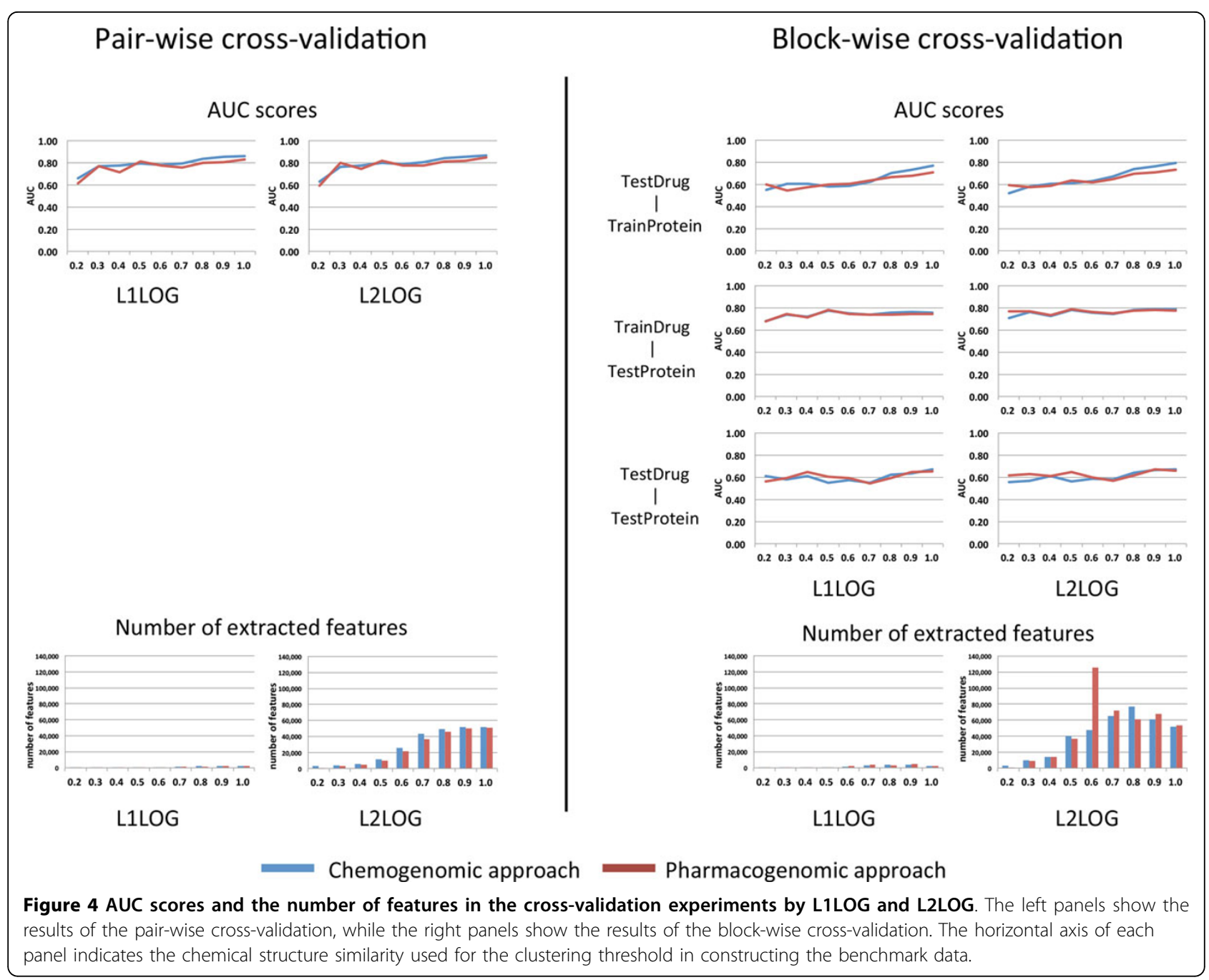

interactions. We computed the total AUC score (the area under the ROC curve) over the five folds.

Figure 4 shows the resulting AUC scores and the number of inferred features by L1LOG and L2LOG based on nine benchmark datasets with different clustering thresholds (see the Method section for more details). It is found that L1LOG is able to infer a smaller number of features, compared with L2LOG in most cases. Interestingly, the prediction accuracy of L1LOG is kept to some extent. Another observation is that the block-wise cross-validation provides lower AUC scores, compared with the pair-wise cross-validation. This result suggests that target prediction for new drugs with no target information and ligand prediction for orphan proteins with no ligand information are quite difficult problems.

Pharmacogenomic approach and chemogenomic approach showed similar behaviors in the pair-wise cross-validation setting, while the two approaches showed different behaviors in the block-wise cross-validation setting. The performance of pharmacogenomic approach was better than that of chemogenomic approach for the benchmark data consisting of structurally different drugs (i.e., in the case of low chemical similarity thresholds). On the other hand, the performance of pharmacogenomic approach was worse than the chemogenomic approach for benchmark data containing many structurally similar drugs (i.e., in the case of high chemical similarity thresholds). For example, the pharmacogenomic approach worked well for the TestDrug-TrainProtein pairs when the chemical similarity threshold is 0.2 , and for the TestDrug-TestProtein pairs when the chemical similarity threshold lies in the range 0.2-0.5.

Figure 5 shows the resulting AUC scores and the number of inferred features for L1LOG-C and L2LOG-C based on nine benchmark datasets. Note that L1LOG-C and L2LOG-C are based on the combination of the tensor product descriptor and individual feature vectors of drugs and target proteins. Similar tendencies exhibited in L1LOG and L2LOG can be observed in L1LOG-C and 


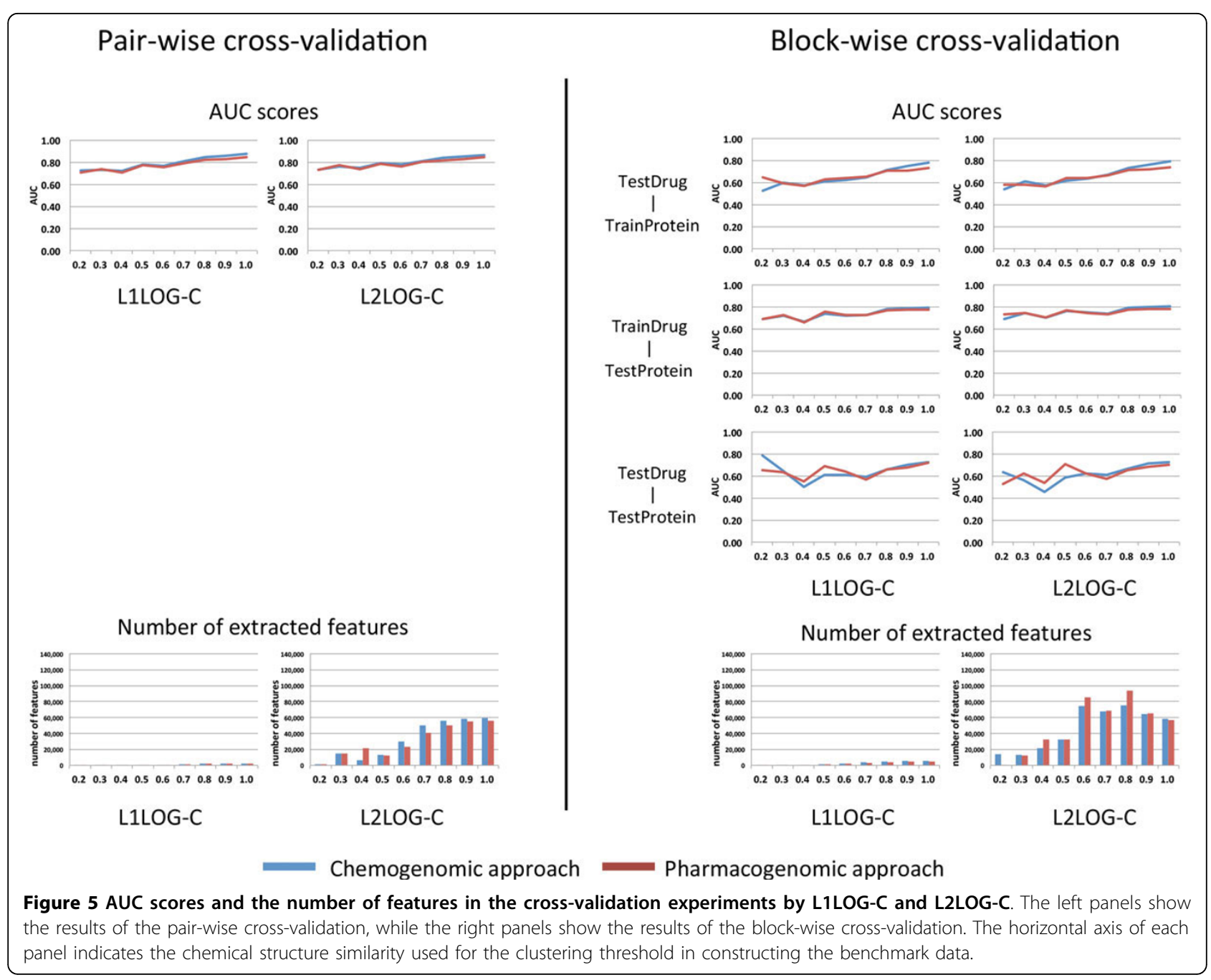

L2LOG-C as well. However, the AUC scores of L1LOG$\mathrm{C}$ and L2LOG-C tend to be higher than those of L1LOG and L2LOG in both the pharmacogenomic approach and the chemogenomic approach. This result suggests that the combination of the tensor product descriptor and individual feature vectors of drugs and target proteins is meaningful for predicting drug-target interactions.

\section{Biological interpretation of side effects and protein domains in the inferred pharmacogenomic features}

We made biological interpretations for drug side effects and protein domains in the inferred pharmacogenomic features. Table 1 shows examples of highly weighted side effect-domain pairs in the pharmacogenomic features inferred by the L1LOG method. Table 2 shows examples of highly weighted protein domains for each side effect inferred by the L1LOG method. Table 3 shows examples of highly weighted side effects for each protein domain inferred by the L1LOG method. The inferred side effect-domain associations suggest potential side effects involving each protein domain and side effects for a wide range of protein families. The results of all inferred pharmacogenomic features in Tables 1, 2, and 3 can be obtained from Additional files 1, 2, and 3 in supplemental materials. Here we discuss some examples in Tables 1, 2, and 3.

7TM_GPCR_Srw, 7TM_GPCR_Srbc, and 7TM_GPCR_ Srsx are the Serpentine type 7TM GPCR chemoreceptors, which are the members of seven-transmembrane G-protein-coupled receptors (7TM GPCRs) that involved in many diseases and are also the target of many modern medicinal drugs. Srw, Srbc, and Srsx are the solo families amongst the superfamilies of chemoreceptors. It is reasonable to find that these three families share some side effects such as mental depression, nasal congestion, and priapism, however it might be meaningful to find that these families have their own specific side effects; shortness of breath, weakness, and ptosis for Srw, labyrinthitis, 
Table 1 Examples of highly weighted pharmacogenomic features inferred by the L1LOG method

\begin{tabular}{ccccc}
\hline Rank & Weight & Side effect & Protein domain ID & Protein domain definition \\
\hline 1 & 330.0000 & anaphylaxis & PF13558 & SbcCD_C (Putative exonuclease SbcCD, C subunit) \\
2 & 330.0000 & tardive dyskinesia & PF10320 & 7TM_GPCR_Srsx (Serpentine type 7TM GPCR chemoreceptor Srsx) \\
3 & 330.0000 & labyrinthitis & PF10316 & 7TM_GPCR_Srbc (Serpentine type 7TM GPCR chemoreceptor Srbc) \\
4 & 330.0000 & shortness of breath & PF02898 & NO_synthase (Nitric oxide synthase, oxygenase domain) \\
5 & 330.0000 & priapism & PF10324 & 7TM_GPC_Srw (Serpentine type 7TM GPCR chemoreceptor Srw) \\
6 & 330.0000 & nasal congestion & PF10324 & 7TM_GPCR_Srw (Serpentine type 7TM GPCR chemoreceptor Srw) \\
7 & 330.0000 & nasal congestion & PF10316 & 7TM_GPCR_Srbc (Serpentine type 7TM GPCR chemoreceptor Srbc) \\
8 & 330.0000 & weakness & PF10324 & 7TM_GPCR_Srw (Serpentine type 7TM GPCR chemoreceptor Srw) \\
9 & 327.1335 & burning sensation & PF10316 & 7TM_GPCR_Srbc (Serpentine type 7TM GPCR chemoreceptor Srbc) \\
10 & 326.7361 & glycosuria & PF00191 & Annexin (Annexin) \\
11 & 325.3147 & shortness of breath & PF10324 & 7TM_GPCR_Srw (Serpentine type 7TM GPCR chemoreceptor Srw) \\
12 & 324.4456 & shortness of breath & PF02931 & Neur_chan_LBD (Neurotransmitter-gated ion-channel ligand binding domain) \\
13 & 320.0000 & anaphylaxis & PF00060 & Lig_chan (Ligand-gated ion channel) \\
14 & 292.0382 & hypoventilation & PF02898 & NF_synthase (Nitric oxide synthase, oxygenase domain) \\
15 & 281.0425 & hypoventilation & PF02931 & Neur_chan_LBD (Neurotransmitter-gated ion-channel ligand binding domain) \\
\hline
\end{tabular}

burning sensation and torticollis for Srbc, and tardive dyskinesia, hyperprolactinemia, and Parkinson for Srsx.

Neur_chan_LBD (Neurotransmitter-gated ion-channel ligand binding domain) is a transmembrane receptorion channel complex that binds specific ligands for rapid transmission of signals at chemical synapses, which includes nicotinic acetylcholine receptor (AchR), glycine receptor, gamma-aminobutyric acid (GABA) receptor, serotonin $5 \mathrm{HT} 3$ receptor, and glutamate receptor. By viewing the side effects on the protein domain level, this domain was shown to be involved in many side effects, such as shortness of breath, respiratory arrest, blindness, hypoventilation, increased salivation, drug dependence, and proctitis. It is understandable that most of these side-effects are shared by NO_synthase (Nitric oxide synthase, oxygenase domain). NO_synthase has isoenzymes eNOS (endothelial NOS) and nNOS (neuronal NOS); the former is the primary signal generator in the control of vascular tone, insulin secretion, and airway tone, and the latter is involved in the development of nervous system.

\section{Novel predictions}

Finally, we conducted a large-scale prediction of unknown interactions of all drugs and all proteins based on the pharmacogenomic features inferred by L1LOG. We learned a predictive model based on all drug-target pairs in the gold standard data, and applied it to all drugs and proteins for which side-effect information and domain information are available. We put the list of the top 1000 predictions in Additional file 4.

\section{Conclusion}

In this paper we made a systematic analysis of the correlation between drug side effects and protein domains, which we call pharmacogenomic features, using binary classifiers with sparse models based on the drug-target interaction network. We showed the usefulness of the inferred pharmacogenomic features for predicting drugtarget interactions. To our knowledge, this work is the first study to relate protein domains with drug side effects on a large scale.

In this study, we used logistic regression as a binary classifier, but other classifiers can be used for the same objective. For example, support vector machine (SVM) is a good candidate for high-performance binary classifier. Actually, we performed the same analysis using SVM in a similar manner as logistic regression, and confirmed that the same tendency in the results can be obtained. The detailed results can be found in Additional files 5 and 6 .

In this study we used side effect profiles of drugs and domain profiles of target proteins in the correlation analysis, but the performance and the biological interpretation depend heavily on the elements in the profiles of drugs and proteins. The method can not extract features which are absent from the predefined descriptors, so the generalization properties of the method could be improved by constructing more meaningful descriptors or using more complete descriptors.

\section{Materials and methods \\ Data \\ Drug-target interactions}

We obtained the information about drug-target interactions from the DrugBank database [18]. The number of interactions in the dataset is 1064 . These interactions involve 413 drugs and 400 target proteins. We used this data set as gold standard data in the cross-validation experiment. 
Table 2 Examples of highly weighted protein domains for each side effect inferred by the L1LOG method

\begin{tabular}{|c|c|c|c|c|}
\hline Rank & Weight & Side effect & Protein domain ID & Protein domain definition \\
\hline \multirow[t]{6}{*}{1} & & anaphylaxis & & \\
\hline & 330.0000 & & PF13558 & SbcCD_C (Putative exonuclease SbcCD, C subunit) \\
\hline & 320.0000 & & PF00060 & Lig_chan (Ligand-gated ion channel) \\
\hline & 115.8250 & & PF08491 & SE (Squalene epoxidase) \\
\hline & 113.4802 & & PF00989 & PAS (PAS fold) \\
\hline & 74.9419 & & PF05790 & C2-set (Immunoglobulin C2-set domain) \\
\hline \multirow[t]{2}{*}{2} & & tardive dyskinesia & & \\
\hline & 330.0000 & & PF10320 & 7TM_GPCR_Srsx (Serpentine type 7TM GPCR chemoreceptor Srsx) \\
\hline \multirow[t]{3}{*}{3} & & labyrinthitis & & \\
\hline & 330.0000 & & PF10316 & 7TM_GPCR_Srbc (Serpentine type 7TM GPCR chemoreceptor Srbc) \\
\hline & 70.4820 & & PF10140 & YukC (WXG100 protein secretion system (Wss), protein YukC) \\
\hline \multirow[t]{5}{*}{4} & & shortness of breath & & \\
\hline & 330.0000 & & PF02898 & NO_synthase (Nitric oxide synthase, oxygenase domain) \\
\hline & 325.3147 & & PF10324 & 7TM_GPCR_Srw (Serpentine type 7TM GPCR chemoreceptor Srw) \\
\hline & 324.4456 & & PF02931 & Neur_chan_LBD (Neurotransmitter-gated ion-channel ligand binding domain) \\
\hline & 100.7863 & & PF10320 & 7TM_GPCR_Srsx (Serpentine type 7TM GPCR chemoreceptor Srsx) \\
\hline \multirow[t]{4}{*}{5} & & priapism & & \\
\hline & 330.0000 & & PF10324 & 7TM_GPCR_Srw (Serpentine type 7TM GPCR chemoreceptor Srw) \\
\hline & 112.9922 & & PF10316 & 7TM_GPCR_Srbc (Serpentine type 7TM GPCR chemoreceptor Srbc) \\
\hline & 2.0630 & & PF00206 & Lyase_1 (Lyase) \\
\hline \multirow[t]{3}{*}{6} & & nasal congestion & & \\
\hline & 330.0000 & & PF10324 & 7TM_GPCR_Srw (Serpentine type 7TM GPCR chemoreceptor Srw) \\
\hline & 330.0000 & & PF10316 & 7TM_GPCR_Srbc (Serpentine type 7TM GPCR chemoreceptor Srbc) \\
\hline \multirow[t]{2}{*}{7} & & weakness & & \\
\hline & 330.0000 & & PF10324 & 7TM_GPCR_Srw (Serpentine type 7TM GPCR chemoreceptor Srw) \\
\hline \multirow[t]{6}{*}{8} & & burning sensation & & \\
\hline & 327.1335 & & PF10316 & 7TM_GPCR_Srbc (Serpentine type 7TM GPCR chemoreceptor Srbc) \\
\hline & 14.2660 & & PF02867 & Ribonuc_red_lgC (Ribonucleotide reductase, barrel domain) \\
\hline & 7.4168 & & PF03522 & KCl_Cotrans_1 (K-Cl Co-transporter type 1 (KCC1)) \\
\hline & 5.7520 & & PF10324 & 7TM_GPCR_Srw (Serpentine type 7TM GPCR chemoreceptor Srw) \\
\hline & 0.3741 & & PF00209 & SNF (Sodium:neurotransmitter symporter family) \\
\hline \multirow[t]{6}{*}{9} & & glycosuria & & \\
\hline & 326.7361 & & PF00191 & Annexin (Annexin) \\
\hline & 145.3436 & & PF08377 & MAP2_projctn (MAP2/Tau projection domain) \\
\hline & 49.9279 & & PF03491 & 5HT_transporter (Serotonin (5-HT) neurotransmitter transporter, N-terminus) \\
\hline & 48.0767 & & PF02222 & ATP-grasp (ATP-grasp domain) \\
\hline & 32.5342 & & PF00698 & Acyl_transf_1 (Acyl transferase domain) \\
\hline \multirow[t]{3}{*}{10} & & hypoventilation & & \\
\hline & 292.0382 & & PF02898 & NO_synthase (Nitric oxide synthase, oxygenase domain) \\
\hline & 281.0425 & & PF02931 & Neur_chan_LBD (Neurotransmitter-gated ion-channel ligand binding domain) \\
\hline
\end{tabular}

\section{Pharmacological and chemical data of drugs}

We obtained the information about side effects of drugs from the SIDER database that accumulates reported side effects from package inserts for marketed drugs [19]. We represented each drug by a 1179-dimensional binary vector in which the presence or absence of each side effect is coded as 1 or 0 .

We obtained the information about chemical structures of drugs from the PubChem database [20]. We represented each drug by an 881-dimensional binary vector in which 881 PubChem substructures are used and the presence or absence of each substructure is coded as 1 or 0 .

\section{Genomic and functional data of target proteins}

We obtained genomic information about target proteins from the UniProt database [21], and obtained the protein domains from the PFAM database [22]. We represented each target protein by a 476-dimensional binary vector in 
Table 3 Examples of highly weighted side effects for each protein domain inferred by the L1LOG method

\begin{tabular}{|c|c|c|c|c|}
\hline Rank & Weight & Protein domain ID & Protein domain definition & Side effect \\
\hline \multirow[t]{3}{*}{1} & & PF13558 & SbcCD_C (Putative exonuclease SbcCD, C subunit) & \\
\hline & 330.0000 & & & anaphylaxis \\
\hline & 41.1178 & & & allergic reaction \\
\hline \multirow[t]{6}{*}{2} & & PF10320 & 7TM_GPCR_Srsx (Serpentine type 7TM GPCR chemoreceptor Srsx) & \\
\hline & 330.0000 & & & tardive dyskinesia \\
\hline & 216.3340 & & & mental depression \\
\hline & 102.3075 & & & regurgitation \\
\hline & 100.7863 & & & shortness of breath \\
\hline & 90.8773 & & & hyperprolactinemia \\
\hline \multirow[t]{6}{*}{3} & & PF10316 & 7TM_GPCR_Srbc (Serpentine type 7TM GPCR chemoreceptor Srbc) & \\
\hline & 330.0000 & & & labyrinthitis \\
\hline & 330.0000 & & & nasal congestion \\
\hline & 327.1335 & & & burning sensation \\
\hline & 266.0200 & & & torticollis \\
\hline & 225.7442 & & & testicular swelling \\
\hline \multirow[t]{6}{*}{4} & & PF02898 & NO_synthase (Nitric oxide synthase, oxygenase domain) & \\
\hline & 330.0000 & & & shortness of breath \\
\hline & 292.0382 & & & hypoventilation \\
\hline & 232.9827 & & & respiratory arrest \\
\hline & 229.9422 & & & increased salivation \\
\hline & 204.4183 & & & blindness \\
\hline \multirow[t]{6}{*}{5} & & PF10324 & 7TM_GPCR_Srw (Serpentine type 7TM GPCR chemoreceptor Srw) & \\
\hline & 330.0000 & & & priapism \\
\hline & 330.0000 & & & nasal congestion \\
\hline & 330.0000 & & & weakness \\
\hline & 325.3147 & & & shortness of breath \\
\hline & 279.9735 & & & ptosis \\
\hline \multirow[t]{6}{*}{6} & & PF00191 & Annexin (Annexin) & \\
\hline & 326.7361 & & & glycosuria \\
\hline & 117.1047 & & & vasculitis \\
\hline & 95.9706 & & & sialadenitis \\
\hline & 79.3155 & & & COPD \\
\hline & 71.9669 & & & choking \\
\hline \multirow[t]{6}{*}{7} & & PF02931 & Neur_chan_LBD (Neurotransmitter-gated ion-channel ligand binding domain) & \\
\hline & 324.4456 & & & shortness of breath \\
\hline & 281.0425 & & & hypoventilation \\
\hline & 244.4901 & & & increased salivation \\
\hline & 228.2349 & & & respiratory arrest \\
\hline & 221.8014 & & & drug dependence \\
\hline \multirow[t]{3}{*}{8} & & PF00060 & Lig-chan (Ligand-gated ion channel) & \\
\hline & 320.0000 & & & anaphylaxis \\
\hline & 19.4911 & & & allergic reaction \\
\hline \multirow[t]{6}{*}{9} & & PF08377 & MAP2_projctn (MAP2/Tau projection domain) & \\
\hline & 251.7744 & & & hyperuricemia \\
\hline & 145.3436 & & & glycosuria \\
\hline & 47.3304 & & & sialadenitis \\
\hline & 44.6506 & & & choking \\
\hline & 36.2648 & & & polydipsia \\
\hline
\end{tabular}




\begin{tabular}{ccc}
\hline 10 & PF14396 CFTR_R (Cystic fibrosis TM conductance regulator (CFTR), regulator domain) & gallbladder disease \\
239.2866 & gynecomastia \\
212.0452 & paronychia \\
156.3952 & prostatism \\
134.2967 & cervical erosion \\
131.3925 & cron \\
\hline
\end{tabular}

which 476 PFAM domains are used and the presence or absence of each domain is coded as 1 or 0 .

\section{Classifiers for drug-target pairs}

We consider the feature extraction problem in the context of drug-target interaction prediction. We represent a pair of drug $D$ and protein $P$ by $(D, P)$. Suppose that we are given a learning set of drug-target pairs $\left(D_{i}, P_{j}\right)(i=1$, $\left.2, \ldots, n_{D} ; j=1,2, \ldots, n_{P}\right)$, where the pairs are known to interact or not, $n_{D}$ is the number of drugs and $n_{P}$ is the number of target proteins in the learning set.

We represent a pair of drug $D$ and protein $P$ by a feature vector $\Phi(D, P)$, and then estimate a function $f(D$, $P)=\boldsymbol{w}^{\mathrm{T}} \Phi(D, P)$ which would predict whether drug-target pair $(D, P)$ is an interacting pair or not. We optimize the weight vector $\boldsymbol{w}$ based on the learning set with label information.

The feature vector of drug $D$ is supposed to be represented as an $M$-dimensional binary vector:

$$
\Phi(D)=\left(d_{1}, d_{2}, \ldots, d_{M}\right)^{\mathrm{T}},
$$

where $d_{k} \in\{0,1\}, k=1, \ldots, M$. For example, $\Phi(D)$ is a profile of side effects or chemical substructures in this study. In the same manner, the feature vector of protein $P$ is supposed to be represented as an $N$-dimensional binary vector:

$$
\Phi(P)=\left(p_{1}, p_{2}, \ldots, p_{N}\right)^{\mathrm{T}}
$$

where $p_{l} \in\{0,1\}, l=1, \ldots, N$. For example, $\Phi(P)$ is a profile of protein domains in this study.

We propose two kinds of feature vectors for each drug-target pair. First, we represent each drug-target pair by the tensor product between $\Phi(D)$ and $\Phi(P)$ as follows:

$$
\begin{aligned}
\Phi(D, P) & =\Phi(D) \otimes \Phi(P) \\
& =\left(d_{1} p_{1}, \ldots, d_{1} p_{N}, \ldots, d_{M} p_{1}, \ldots, d_{M} p_{N}\right)^{\mathrm{T}},
\end{aligned}
$$

where $\Phi(D, P)$ is an $(M \times N)$-dimensional feature vector. We refer to the feature vector as "tensor product descriptor" in this study. This tensor product descriptor is similar to that in the previous work [12].

Second, we represent each drug-target pair by the combination of the tensor product descriptor
$\Phi(D) \otimes \Phi(P)$ and individual feature vectors $\Phi(D)$ and $\Phi(P)$ as follows:

$$
\begin{aligned}
\Phi_{C}(D, P) & =\left[(\Phi(D) \otimes \Phi(P))^{\mathrm{T}}, \Phi(D)^{\mathrm{T}}, \Phi(P)^{\mathrm{T}}\right]^{\mathrm{T}} \\
& =\left(d_{1} p_{1}, \ldots, d_{1} p_{N}, \ldots, d_{M} p_{1}, \ldots, d_{M} p_{N}, d_{1}, d_{2}, \ldots, d_{M}, p_{1}, p_{2}, \ldots, p_{N}\right)^{\mathrm{T}},
\end{aligned}
$$

where $\Phi_{C}(D, P)$ is an $(M \times N+M+N)$-dimensional binary vector. We refer to the feature vector as "combined descriptor" in this study.

In this study we use logistic regression as a binary classifier to predict whether a drug $D$ interacts with a target protein $P$ or not. The predictive model is usually learnt by minimizing the loss function with $L_{2}$-regularization. However, $L_{2}$-regularization tends to keep most weight elements to be non-zeros, which makes it difficult to interpret features from the resulting weight vector. Another possible solution is to use $L_{1}$-regularization that tends to make most weight elements to be zeros, which makes it easier to interpret features from the resulting weight vector. Therefore, we introduce a logistic regression model with $L_{1}$-regularization.

Suppose that we have a learning set of drug-target pairs and interaction labels

$\left(\Phi\left(D_{i}, P_{j}\right), y_{i j}\right), y_{i j} \in\{+1,-1\}\left(i=1,2, \ldots, n_{D} ; j=1,2\right.$, $\left.\ldots, n_{P}\right)$, where $n_{D}$ is the number of drugs and $n_{P}$ is the number of target proteins in the learning set. The weight vector $\boldsymbol{w}$ of the linear logistic regression is usually learned with $L_{2}$-regularization as follows:

$$
\min _{w}\|w\|_{2}+C \sum_{i=1}^{n_{D}} \sum_{j=1}^{n_{P}} \log \left(1+\exp \left(-y_{i j} w^{\mathrm{T}} \Phi\left(D_{i}, P_{j}\right)\right)\right)
$$

where $\|\cdot\|_{2}$ is $L_{2}$ norm (the sum of squared values) and $C$ is a regularization parameter to control the penalty.

To induce sparsity in the model, the weight vector $w$ of the linear logistic regression is learned with $L_{1}$-regularization as follows:

$$
\min _{w}\|w\|_{1}+C \sum_{i=1}^{n_{D}} \sum_{j=1}^{n_{P}} \log \left(1+\exp \left(-y_{i j} w^{\mathrm{T}} \Phi\left(D_{i}, P_{j}\right)\right)\right)
$$

where $\|\cdot\|_{1}$ is $L_{1}$ norm (the sum of absolute values) and $C$ is a regularization parameter to control the sparsity. We examine various values $(0.0001,0.001,0.01,0.1$, $1,10,100,1000,10000)$ for the hyper parameter $C$, and 
select the value that gave the highest AUC score in the cross-validation experiment.

In practice, we consider applying the logistic regression with the tensor product descriptor $\Phi(D, P)$ and with the combined descriptor $\Phi_{C}(D, P)$. We refer to $L_{1^{-}}$ regularized logistic regression with the tensor product descriptor as "L1LOG," and $L_{2}$-regularized logistic regression with the tensor product descriptor as "L2LOG," respectively. We refer to $L_{1}$-regularized logistic regression with the combined descriptor as "L1LOGC," and $L_{2}$-regularized logistic regression with the combined descriptor as "L2LOG-C," respectively.

\section{Cross-validation experiments for benchmark data}

There are two scenarios for drug-target interaction prediction from practical viewpoints. The first scenario is that we have drugs with target information and proteins with ligand information, and the goal is to additionally detect missing interactions between the drugs and the proteins. The second scenario is that we have drugs with no target information and protein with no ligand information, and the goal is to find all potential target proteins of the drugs and all potential ligands of target proteins. To simulate the above two scenarios in the 5fold cross-validation experiment, we consider two different settings: pair-wise cross-validation and block-wise cross-validation.

The pair-wise cross-validation consists of the following procedures: First, we split all drug-target pairs in the gold standard set into five subsets of all drug-target pairs in an independent manner. Second, we regard each subset of drug-target pairs as a test set, and regard the other four subsets of drug-target pairs as a training set. Third, we optimize a predictive model based on drug-target pairs in the training set. Finally, we apply the predictive model to drug-target pairs in the test set. Note that drug-target pairs are considered independent of each other, so drugs and target proteins in test pairs are overlapped with those in the training set to some extent.

The block-wise cross-validation consists of the following procedures: First, we split drugs and target proteins in the gold standard set into five subsets of drugs and five subsets of target proteins. Second, we regard each subset of drugs (resp. proteins) as test drugs (resp. test proteins), and use the other four subsets of drugs as training drugs (resp. training proteins). Third, we optimize a predictive model based on drug-target pairs consisting of training drugs and training proteins. Finally, we compute the prediction scores for three types of drug-target pairs: test drugs v.s. training target proteins (referred to as "TestDrug-TrainProtein"), training drugs v.s. test target proteins (referred to as "TrainDrug-TestProtein"), and test drugs v.s. test target proteins (referred to as "TestDrug-TestProtein"). Note that drugs and proteins in test pairs are not completely different from those in the training set. Thus, the prediction problem in the blockwise setting is more difficult than that in the pair-wise setting.

The gold standard data contain many drugs which were chemically and structurally almost identical, because they were derived from the same lead compound. If these identical drugs were divided into a training set and a test set, the prediction in the cross-validation experiment would be trivial. To avoid overestimation of the prediction accuracy, we perform a grouping of similar drugs based on their chemical structures and use only drugs which are chemically and structurally different to some extent, following a previous work [10]. First, we carry out a clustering of all drugs based on Tanimoto coefficient (Jaccard coefficient) [23] of chemical fingerprints using average linkage algorithm. Second, we cluster drugs with high Tanimoto coefficients into the same cluster, and selected one representative drug within each cluster. Third, we construct a set of drugs with low Tanimoto coefficients. Finally, we prepare nine sets of benchmark data consisting of representative drugs by varying the clustering threshold little by little (e.g., from 0.2 to 1.0 by 0.1 ) on the dendrogram. When the clustering threshold is 0.1 , the number of drug clusters is only 3 in our data, so it is not possible to test the clustering threshold of 0.1 in the 5 -fold cross-validation.

\section{Additional material}

Additional file 1: Extracted side effects and protein domains of
L1LOG.
Additional file 2: Extracted protein domains for each side effect of
L1LOG.
Additional file 3: Extracted side effects for each protein domain of
L1LOG.
Additional file 4: The list of novel drug-target predictions of L1LOG.
Additional file 5: AUC scores and the number of features in the
pair-wise and block-wise cross-validation experiments by L1LOG,
L2LOG, L1SVM, and L2SVM.
Additional file 6: AUC scores and the number of features in the
pair-wise and block-wise cross-validation experiments by L1LOG-C,
L2LOG-C, L1SVM-C, and L2SVM-C.

Competing interests

The authors declare that they have no competing interests.

Authors' contributions

$\mathrm{HI}$ tested the performance of the methods and drafted the manuscript. SM prepared the datasets and made biological interpretations of the results. YT implemented the algorithm of the methods. MK made biological

interpretations of the results and drafted the manuscript. SG drafted the manuscript. YY directed the work, and drafted the manuscript. All authors read and approved the final manuscript. 


\section{Acknowledgements}

This work was supported by MEXT/JSPS KAKENHI Grant Numbers 25108714 24700140, and 25700029. This work was also supported by the Program to Disseminate Tenure Tracking System, MEXT, Japan, and Kyushu University Interdisciplinary Programs in Education and Projects in Research Development.

\section{Declarations}

The publication cost for this work was supported by JSPS Kakenhi 25700029. This article has been published as part of BMC Systems Biology Volume 7 Supplement 6, 2013: Selected articles from the 24th International Conference on Genome Informatics (GIW2013). The full contents of the supplement are available online at http://www.biomedcentral.com/bmcsystbiol/supplements/ $7 / 56$.

\section{Authors' details}

'Division of System Cohort, Multi-scale Research Center for Medical Science, Medical Institute of Bioregulation, Kyushu University, 3-1-1 Maidashi, Higashiku, Fukuoka, Fukuoka 812-8582, Japan. ${ }^{2}$ Bioinfromatics Center, Institute for Chemical Research, Kyoto University, Gokasho, Uji, Kyoto 611-0011, Japan. ${ }^{3}$ PRESTO, Japan Science and Technology Agency, Kawaguchi, Saitama 3320012, Japan. ${ }^{4}$ Institute for Advanced Study, Kyushu University, 6-10-1, Hakozaki, Higashi-ku, Fukuoka, Fukuoka 812-8581, Japan.

Published: 13 December 2013

\section{References}

1. Whitebread S, Hamon J, Bojanic D, Urban L: Keynote review: In vitro safety pharmacology profiling: an essential tool for successful drug development. Drug discovery today 2005, 10(21):1421-1433.

2. Yamanishi Y, Araki M, Gutteridge A, Honda W, Kanehisa M: Prediction of drug-target interaction networks from the integration of chemical and genomic spaces. Bioinformatics 2008, 24(13):i232-i240.

3. Faulon J, Misra M, Martin S, Sale K, Sapra R: Genome scale enzymemetabolite and drug-target interaction predictions using the signature molecular descriptor. Bioinformatics 2008, 24:225-233.

4. Jacob L, Vert J: Protein-ligand interaction prediction: an improved chemogenomics approach. Bioinformatics 2008, 24:2149-2156.

5. Bleakley $K$, Yamanishi $Y$ : Supervised prediction of drug-target interactions using bipartite local models. Bioinformatics 2009, 25(18):2397-2403.

6. Keiser MJ, Setola V, Irwin JJ, Laggner C, Abbas Al, Hufeisen SJ, Jensen NH, Kuijer MB, Matos RC, Tran TB, et al: Predicting new molecular targets for known drugs. Nature 2009, 462(7270):175-181.

7. van Laarhoven T, Nabuurs SB, Marchiori E: Gaussian interaction profile kernels for predicting drug-target interaction. Bioinformatics 2011, 27(21):3036-3043.

8. Campillos M, Kuhn M, Gavin AC, Jensen L, Bork P: Drug target identification using side-effect similarity. Science 2008, 321(5886):263-266.

9. Yamanishi $Y$, Kotera M, Kanehisa M, Goto S: Drug-target interaction prediction from chemical, genomic and pharmacological data in an integrated framework. Bioinformatics 2010, 26(12):i246-i254.

10. Takarabe M, Kotera M, Nishimura Y, Goto S, Yamanishi Y: Drug target prediction using adverse event report systems: a pharmacogenomic approach. Bioinformatics 2012, 28(18):i611-i618.

11. Yamanishi Y, Pauwels E, Saigo H, Stoven V: Extracting Sets of Chemical Substructures and Protein Domains Governing Drug-Target Interactions. Journal of Chemical Information and Modeling 2011, 51:1183-1194.

12. Tabei $Y$, Pauwels $E$, Stoven $V$, Takemoto $K$, Yamanishi $Y$ : Identification of chemogenomic features from drug-target interaction networks using interpretable classifiers. Bioinformatics 2012, 28(18):i487-i494.

13. Takigawa I, Tsuda K, Mamitsuka H: Mining Significant Substructure Pairs for Interpreting Polypharmacology in Drug-Target Network. PloS one 2011, 6:e16999.

14. Mizutani S, Pauwels E, Stoven V, Goto S, Yamanishi Y: Relating drugprotein interaction network with drug side effects. Bioinformatics 2012, 28(18):i522-i528.

15. Yamanishi $Y$, Pauwels E, Kotera M: Drug Side-Effect Prediction Based on the Integration of Chemical and Biological Spaces. Journal of chemical information and modeling 2012, 52(12):3284-3292.

16. Lounkine E, Keiser MJ, Whitebread S, Mikhailov D, Hamon J, Jenkins JL, Lavan $\mathrm{P}$, Weber E, Doak AK, Côté S, et al: Large-scale prediction and testing of drug activity on side-effect targets. Nature 2012, 486(7403):361-367.

17. Kuhn M, Al Banchaabouchi M, Campillos M, Jensen LJ, Gross C, Gavin AC, Bork P: Systematic identification of proteins that elicit drug side effects. Molecular systems biology 2013, 9 .

18. Knox C, Law V, Jewison T, Liu P, Ly S, Frolkis A, Pon A, Banco K, Mak C, Neveu $V$, et al: DrugBank 3.0: a comprehensive resource for ?omics? research on drugs. Nucleic acids research 2011, 39(suppl 1):D1035-D1041.

19. Kuhn M, Campillos M, Letunic I, Jensen LJ, Bork P: A side effect resource to capture phenotypic effects of drugs. Molecular systems biology 2010, 6 .

20. Chen B, Wild D, Guha R: PubChem as a source of polypharmacology. $J$ Chem Inf Model 2009, 49:2044-2055.

21. Consortium TU: The Universal Protein Resource (UniProt) in 2010. Nucleic Acids Res 2010, 38:D142-D148.

22. Finn R, Tate J, Mistry J, Coggill P, Sammut J, Hotz H, Ceric G, Forslund K, Eddy S, Sonnhammer E, Bateman A: The Pfam protein families database. Nucleic Acids Res 2008, 36:D281-D288.

23. Tanimoto T: An elementary mathematical theory of classification and prediction 1958, International Business Machines Corporation.

doi:10.1186/1752-0509-7-S6-S18

Cite this article as: Iwata et al.: Inferring protein domains associated with drug side effects based on drug-target interaction network. BMC Systems Biology 2013 7(Suppl 6):S18.

\section{Submit your next manuscript to BioMed Central and take full advantage of:}

- Convenient online submission

- Thorough peer review

- No space constraints or color figure charges

- Immediate publication on acceptance

- Inclusion in PubMed, CAS, Scopus and Google Scholar

- Research which is freely available for redistribution

Submit your manuscript at www.biomedcentral.com/submit
C Biomed Central 